\title{
Research on Winter Tea Application and Promotion Value
}

\author{
Wu Yan*, Zhangzhi Ge \\ University of Science and Technology of China, Hefei, China \\ Email: *syc16@mail.ustc.edu.cn
}

How to cite this paper: Yan, W., \& Ge, Z. Z. (2020). Research on Winter Tea Application and Promotion Value. Modern Economy, 11, 817-828.

https://doi.org/10.4236/me.2020.114061

Received: February 23, 2020

Accepted: April 13, 2020

Published: April 16, 2020

Copyright $\odot 2020$ by author(s) and Scientific Research Publishing Inc. This work is licensed under the Creative Commons Attribution International License (CC BY 4.0).

http://creativecommons.org/licenses/by/4.0/

\section{(c) (i) Open Access}

\begin{abstract}
This paper researches the application prospect and promotion value of winter tea. The research status of tea polysaccharides, tea polyphenols and catechins, the main constituents of winter tea, were analyzed. In addition, the economic value and social benefit value of winter tea were analyzed. The results show that winter tea can be used as a raw material for extracting tea polysaccharides for the production and $\mathrm{R} \& \mathrm{D}$ of basic substances for the healthy foods and medicines for lower blood sugar, blood lipids, increase immunity and delay aging; winter tea also has broad application prospects and value in biopharmaceuticals, skin care products and food; winter tea can increase the production and income of tea farmers and tea companies. Responding to the Chinese government's strategy to fight poverty, "cold bubble" winter tea is suitable for the consumption habits of young people and residents of countries along the "Belt and Road". Therefore, winter tea has higher application and promotion value.
\end{abstract}

\section{Keywords}

Winter Tea, Tea Polysaccharide, Fight Poverty, Belt and Road

\section{Introduction}

Traditional tea is mainly made from the buds of spring tea trees, summer tea trees and autumn tea trees. The use and attention to the fresh leaves of winter tea trees is relatively small, resulting in the waste of fresh leaves of winter tea trees. The fresh leaves of winter tea trees are generally called thick old leaves. After picking the thick old leaves repeatedly, fermentation and fermentation can produce a brand-new tea called winter tea. Due to the low temperature in winter, the macromolecular sugars in the tea tree will be hydrolyzed to small molecular sugars to increase the concentration of the cell fluid, reduce the freezing point, 
and avoid freezing damage. Therefore, the content of nutrients and sugars in the winter tea is high.

At present, the research on tea mainly focuses on common tea categories such as green tea and black tea, and there are few studies on this kind of thick and old leaves in winter. Ren et al. (2009) expounded the research progress on the extraction of tea polyphenols, caffeine, amino acids, polysaccharides and their health effects from the functional components of green tea. Yang et al. (2009) expounded the research status of green tea extracts, and reviewed its main components, extraction methods, and reviewed its main physiological activity, application and safety evaluation, and prospected its development prospects. Ma et al. (2003) analyzed the content of inorganic elements in Chinese famous green tea and its current status. Zhu et al. (2008) studied the development status of Chinese green tea industry. Jiang et al. (2018) analyzed the current situation and prospects of the black tea industry. Zhu et al. (2015) studied the research status of the black tea fermentation process. Sharma et al. (2020) believed that tea polyphenols theaflavin could be used as anti-Oxidants and potential copper chelators. Chen et al. (2009) studied the antioxidant properties of active ingredients in black tea, green tea, and oolong tea. Hou et al. (2005) believe that black tea has higher tea polyphenol content and strong antioxidant properties. This article will study the application and promotion value of winter tea by analyzing the research status of the main constituents of winter tea and the economic and social effects of winter tea.

\section{Winter Tea}

\subsection{Winter Tea Definition}

China is one of the origins of tea and has a long history of tea production. China has the world's largest tea plantation area and the world's largest tea production. After thousands of years of evolution, tea has formed six widely recognized types of tea in the industry, namely green tea, black tea, oolong tea, black tea, yellow tea, and white tea. Each type of tea has its own suitable picking season, special craftsmanship, and distinctive contents. However, the production of these six major types of tea has the problem of limited production season, and never picking fresh leaves in winter, resulting in a large waste of fresh leaf resources.

Winter tea refers to the thick and old leaves picked from tea trees in $25^{\circ}-32^{\circ}$ north latitude of Xiuning, Yuexi in southern Anhui and Guizhou in southwest after December or the first snow every year, twisting and fermenting (Yuan et al., 2019). Winter tea is similar to autumn tea, the most prominent feature is its high aroma, even better than autumn tea. When the solar terms reach frost, the temperature gradually decreases and the weather becomes drier. At this time, the tea buds grow very slowly, and even dormant and suspend growth. At this time, the tea buds reduced the amount of polyphenols synthesized and metabolized in short days and low temperatures. To cope with the cold, the macromolecular 
sugars in tea will also be hydrolyzed to small molecular sugars to increase the concentration of cell fluid, reduce the freezing point, and avoid freezing damage. Therefore, the content of nutrients and sugars in winter tea is high. The fresh leaves of winter tea trees are generally considered to be coarse old tea leaves. The older the tea leaves, the higher the content of tea polysaccharides. At present, the waste of tea resources in China is relatively serious. Generally, only spring tea or a small amount of autumn tea is used. A large amount of summer tea and pruned branches and leaves are not used.

\subsection{Winter Tea Making Process}

The picked thick old fresh leaves are cleaned with clear water to remove the heavy metals indicated by the fresh leaves. The specific process (Wang et al., 2019) is as Figure 1.

\subsection{Winter Tea Main Ingredients Detection}

The thick old fresh leaves picked in Jinzhai County, Anhui Province at the end of December 2019 were washed, and then killed by a roller at $280^{\circ} \mathrm{C}$ for $40 \mathrm{~s}$, hot-rolled for $30 \mathrm{~min}, 30^{\circ} \mathrm{C}$ fermentation for $24 \mathrm{~h}$, a twister for $15 \mathrm{~min}, 30^{\circ} \mathrm{C}$ secondary fermentation for $24 \mathrm{~h}$, fried The green tea was fried 3 times and baked at $110^{\circ} \mathrm{C}$ for 1 hour to obtain winter tea (Wang et al., 2019). Take $500 \mathrm{~g}$ of prepared winter tea to test the composition and content of winter tea as shown in Table 1.

As can be seen from Table 1, the main components of winter tea are tea polysaccharides, tea polyphenols, catechins, caffeine, theobromine, theanine, linalool, ionone, zinc, selenium, fluorine, and the like.

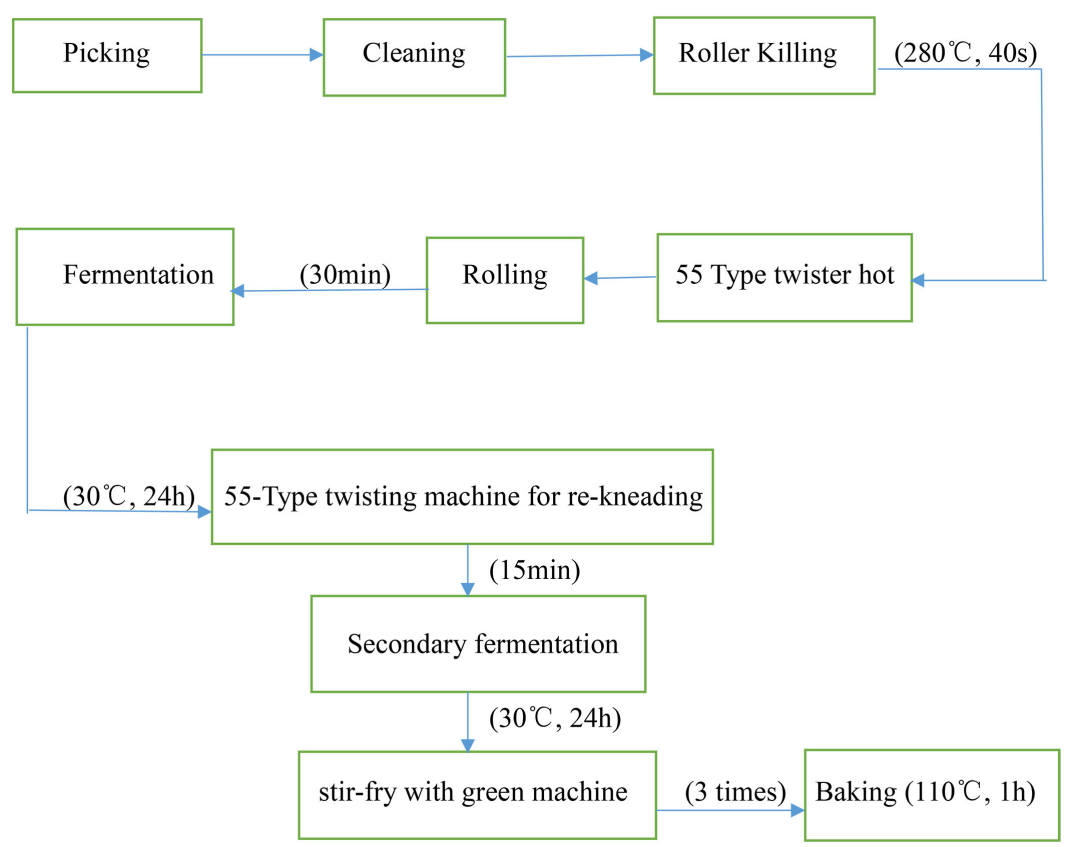

Figure 1. Winter tea preparation process flow chart. 
Table 1. Winter tea main ingredients content table.

\begin{tabular}{ccccc}
\hline $\begin{array}{c}\text { Serial } \\
\text { number }\end{array}$ & Component detected & unit & Detection method & content/\% \\
\hline 1 & Tea polysaccharide & $\mathrm{g} / 100 \mathrm{~g}$ & SN/T 4260-2015 & 2.86 \\
2 & Tea polyphenols & $\%$ & GB/T 8313-2008 & 15.9 \\
3 & Catechin & $\%$ & GB/T 8313-2018 & 37.8 \\
4 & Caffeine & $\%$ & GB/T 8312-2013 & 0.9 \\
5 & Theobromine & $\%$ & GB/T 8312-2013 & 0.009 \\
6 & Theanine & $\mathrm{g} / \mathrm{kg}$ & GB/T 23193-2017 & 1.37 \\
7 & Zinc & $\mathrm{mg} / \mathrm{kg}$ & GB 5009.14-2017 & 30 \\
8 & selenium & $\mathrm{mg} / \mathrm{kg}$ & GB 5009.14-2017 & 0.018 \\
9 & fluorine & $\mathrm{mg} / \mathrm{kg}$ & GB 19965-2005 & 183 \\
10 & Linalool & $\%$ & $\begin{array}{c}\text { Analysis of Aroma Components of } \\
\text { Maojian Tea Based on SPME-MS }\end{array}$ & 0.539 \\
11 & Ionone & $\%$ & $\begin{array}{c}\text { Analysis of Aroma Components of } \\
\text { Maojian Tea Based on SPME-MS }\end{array}$ & 0.633 \\
\hline
\end{tabular}

Usually the tea polysaccharide content of black tea is $0.5 \%-1 \%$, the tea polysaccharide content of green tea is $1 \%-1.5 \%$, and the tea polysaccharide content of winter tea obtained this time is $2.86 \%$. It is a common tea made from tender buds such as black tea and green tea Class 2 - 3 times. The content of tea polyphenols in green tea is $15 \%-30 \%$, and the content of tea polyphenols in winter tea is $15.9 \%$, which is far lower than that of green tea.

\section{Research Status of Main Components of Winter Tea}

\subsection{Tea Polysaccharide Research Status}

Tea polysaccharide is a complex polysaccharide with physiological activity, a natural active substance with great potential for development. Its functional research has always been the focus of researchers. A large number of studies have shown that tea polysaccharides have the effects of lowering blood sugar, lowering blood lipids, anticoagulation, thrombosis, antioxidant and regulating immunity (Yu et al., 2008).

Yu et al. (2010) continuously administered Anji white tea polysaccharide to mice with norepinephrine-induced hyperglycemia and tetraoxopyrimidine-induced diabetic mice for 14 days, and then took blood to measure the blood glucose level. The results showed that Anji white tea polysaccharide can significantly reduce norepinephrine Adrenaline-induced blood glucose levels in diabetic mice, indicating that Anji white tea polysaccharides have a significant effect in alleviating the decrease in glucose tolerance in diabetic mice, reaching the therapeutic level of the drug group. Zhou et al. (2009) studied that Pu'er tea polysaccharide can promote the weight recovery of diabetic mice, and can effectively reduce the blood glucose value of diabetic mice. The high dose of Pu'er tea polysaccharide $(160 \mathrm{mg} / \mathrm{kg})$ has a better effect than the low dose of Pu'er tea Po- 
lysaccharide $(80 \mathrm{mg} / \mathrm{kg})$ on lowering blood sugar. He et al. (2007) observed the inhibitory effect of tea polysaccharide on $\alpha$-amylase activity in vitro, and its effect on normal rats and tetraoxopyrimidine of blood glucose in diabetic rat models, by using 3,5-dinitrosalicylic acid (DNS) colorimetry. The result shows that polysaccharide can reduce fasting blood glucose and postprandial blood glucose in diabetic rats caused by tetraoxopyrimide, and its hypoglycemic mechanism may be related to its inhibition of $\alpha$-amylase activity. Chen et al. (2010) observed the anti-diabetic activity of water-soluble tea polysaccharide conjugate (TPC-W) and alkali-soluble tea polysaccharide conjugate (TPC-A) in non-obese diabetic (NOD) mice as experimental subjects. The results showed that TPC-W and TPC-A can inhibit spontaneous diabetes in NOD mice. Wang et al. (2010) sulfated NTPS and ATPS with pyridine sulfonic acid method to obtain NTPS-S and ATPS-S. The comparative study on the hypoglycemic effect of mice showed that sulfated tea polysaccharide can significantly improve mice hypoglycemic activity.

Chen et al. (2016)'s research showed that tea polysaccharide is a natural antioxidant. Wang et al. (2013) considered that crude tea polysaccharides have stronger antioxidant activity than tea polysaccharide components, and EGCG significantly enhanced the ability of tea polysaccharide components to scavenge DPPH free radicals, and could significantly improve the reducing ability of tea polysaccharide components. At the same time, it can synergistically improve the antioxidant activity of TPS components. Zhang et al. (2011) explored the regulation and mechanism of oolong tea polysaccharides (OTPS) on blood lipids in high-fat model mice. the results showed that compared with the high-fat model control group, serum total cholesterol (TC) and low-density lipids Protein (LDL-C), serum and liver MDA, liver index showed varying degrees of decline, and thymus index, spleen index, serum and liver SOD activity increased, liver lesions were reduced to a certain extent, indicating that oolong tea polysaccharide has a certain Regulate blood lipids, improve body immunity and antioxidant effect.

Sun et al. (2013) studied that tea polysaccharide of Qimen black tea has antioxidative and liver-protective effects on oxidative damage induced by carbon tetrachloride in mice. Wang et al. (2014) considered that tea polysaccharides (ZTPs) extracted from selenium-rich Ziyang green tea could protect the liver. ZTPS has significant scavenging effects on DPPH, $\mathrm{OH}$, and $\mathrm{O}_{2}$, and has strong antioxidative effect in vitro. The effect was further confirmed by inhibiting $\mathrm{CCl} 4$ induced liver oxidative damage in mice. Shen et al. (2007) gave different doses of tea polysaccharides to sarcoma S180 tumor-bearing mice. The results showed that tea polysaccharides had an inhibitory effect on tumor growth. Its tumor suppressing activity may be related to its immune enhancing effect.

\subsection{Research Status of Tea Polyphenols}

Tea polyphenol (GTP) is a complex of polyhydroxy phenolic compounds in tea. It is composed of more than 30 phenolic substances. Its main component is ca- 
techin and its derivatives. It is the main chemical component in tea with health functions. With a variety of physiological activities such as anti-oxidation, anti-radiation, anti-aging, lowering blood lipids, lowering blood sugar, and inhibiting bacteria and enzymes (Wang, 2018).

Jung et al. (2013) considered that the biologically active components of microencapsulated green tea extracts (mainly tea polyphenols) can effectively reduce the adverse characteristics of myocardial fibrosis, reduce uric acid levels, and increase liver catalase activity, helping The vascular system is protective. Zhao (2008) showed that oral tea polyphenols can effectively protect brain tissue from neuronal death caused by 6-OHDA damage. Helieh et al. (2010) through experimental studies believe that tea polyphenols can cause tumor cell apoptosis and lysis to treat and prevent tumor diseases through Fas-related death domain protein-dependent mode. Li et al. (2003) inoculated ewis lung cancer C5 7BL/6J mouse model experiments, studying that tea polyphenols can significantly reduce the serum MDA content of tumor-bearing mice, increase SOD and GSH Px activity to inhibit the growth of Lewis lung cancer.

Studies by Yu et al. (2012) showed that tea polyphenols of Anji white tea have a certain reducing power, which has a significant inhibitory effect on red blood cell oxidative hemolysis and oxidative hemolysis caused by $\mathrm{H}_{2} \mathrm{O}_{2}$, followed by $\mathrm{Fe}^{2+}$ complexing ability and the effect of $-\mathrm{OH}$ removal is relatively weak, indicating that tea polyphenols of Anji white tea can be used as potential natural antioxidants in the food and pharmaceutical industries. Peng et al. (2003) mixed tea polyphenol with influenza virus A3 and inoculated MDCK cells. In the concentration range of $3.12-50 \mu \mathrm{g} / \mathrm{ml}$, the number of MDCK cells increased, indicating that tea polyphenols have a direct inactivation effect on influenza virus A3. In addition, tea polyphenols were given after virus infection. In the concentration range of $6.25-50 \mu \mathrm{g} / \mathrm{ml}$, the number of MDCK cells could be significantly increased, indicating that tea polyphenols have a therapeutic effect on influenza virus A3 and can significantly inhibit the reproduction of influenza virus A3, indicating that tea polyphenols have an anti-influenza virus A3 effect in vitro.

\subsection{Catechin Research Status}

Catechin is a type of phenolic active substance extracted from natural plants such as tea. A large number of experimental studies have found that tea catechin and related constituents can inhibit the proliferation of cancer cells such as intestinal cancer, liver cancer, lung cancer, gastric cancer, breast cancer and prostate, promoting the apoptosis of related cancer cells (Crespy \& Wiliamson, 2004).

Zhang \& Yang (2003) studied that catechin monomers have obvious inhibition and induce apoptosis effects on prostate cancer cells. Yang et al. (2006) studied that catechin can inhibit the proliferation of prostate cancer cells, induce apoptosis of prostate cancer cells, and regulate key enzymes in the formation of 
prostate cancer. Guo et al. (2013) research that catechins can inhibit the expression of TGF- $\beta 1$, TNF- $\alpha$ protein to reduce the inflammation response of early radiation-induced lung injury, to prevent and treat acute lung injury. Hu \& Yang (2001) studied that tea catechins (including EGCG, ECG and EGC) can promote the apoptosis of leukemia cancer cells, promote the generation of free radicals, and inhibit the antioxidant enzyme activity of cancer cells.

Zheng et al. (2005) thought that tea catechins could inhibit the proliferation of influenza A and B viruses. Kao et al. (2006) considered that tea catechins have the effects of weight loss and hypoglycemia, having health benefits for obesity and diabetes.

\section{The Significance of Winter Tea Promotion}

\subsection{As a Raw Material for Extracting Tea Polysaccharides}

Winter tea is mainly made of coarse and old tea leaves with high content of related components, mainly containing tea polysaccharides, tea polyphenols, catechins, and theanine. The content of tea polysaccharides is 3-5 times that of other ordinary tea (Mai et al., 2019).

Winter tea can be used as a raw material for tea polysaccharides. It has a very high cost performance and can greatly reduce production costs. Clinical trials and related studies have shown that tea polysaccharides have the effects of lowering blood sugar, lowering blood lipids, anticoagulation, thrombosis, antioxidation and regulating immunity (Shi et al., 2018).

With the promotion of modern material life, obesity, hyperglycemia, hyperlipidemia and other related diseases are increasing. Tea polysaccharides can be used as basic substances to make related oral agents, medicaments or related functional foods such as hypoglycemic and hypolipidemic drugs, which can prevent and treat symptoms and diseases related to hyperglycemia and hyperlipidemia. The medicine based on tea polysaccharide mainly acts on the molecule. It does not enter the inside of the cell and its side effects are relatively small. So it has high safety and is worthy of application and promotion. Aging is an irreversible change in living organisms. It makes the human body's resistance gradually weaken, eventually leading to the decline of human organs and functions. In addition, aging will increase the risk of cardiovascular and cerebrovascular diseases, dementia, diabetes and cancer-related diseases (Martin, 2011). Chinese aging population is getting higher and higher and the base of aging population is large, which will face severe pressure on medical care for the aging population.

\subsection{Winter Tea's Application Prospects in Biopharmaceuticals, Skin Care Products and Food}

Winter tea mainly contains tea polysaccharides, tea polyphenols, catechins, and theanine. Tea polyphenols in winter tea have various physiological activities such as anti-oxidation, anti-radiation, anti-aging, lowering blood lipids, lowering blood sugar, and inhibiting bacteria and enzymes. Not only that, catechins 
and related constituents in winter tea can inhibit the proliferation of cancer cells such as intestinal cancer, liver cancer, lung cancer, gastric cancer, breast cancer and prostate, and promote the apoptosis of related cancer cells (Liu et al., 2014; Kim et al., 2013). Winter tea powder can be extracted from winter tea as a basic substance for the prevention and treatment of cancer cancers such as intestinal cancer, liver cancer, lung cancer, gastric cancer, breast cancer, and prostate cancer, so it has huge market prospects and application value. In addition, winter tea has anti-oxidant, anti-radiation, anti-aging and other physiological activities, so it can be used in the production of related health products, skin care products and food such as winter tea tablets, winter tea nutrition tablets, winter tea masks, winter tea perfumes, winter tea chocolates, winter tea biscuits, etc. Therefore, the intensive processing of winter tea in biopharmaceuticals, skin care products and food also has a broad market and prospects.

\subsection{Responding to National Poverty Alleviation and the "Belt and Road" Strategy}

Chinese tea is mainly divided into six major types of tea, including spring tea, summer tea, and autumn tea. The spring tea, summer tea, and autumn tea account for $40 \%-50 \%, 10 \%-30 \%$ and $20 \%-40 \%$ of the annual output, respectively (Chen, 2014). Generally, the tea garden is trimmed after the end of autumn, and the tea will be picked in the coming spring. Tea tree leaves in winter are generally considered to be coarse old tea leaves and are not suitable for processing into better quality tea leaves. Therefore, the thick and old leaves in winter are not used, resulting in a waste of resources. Using thick old leaves in winter to make winter tea can make full use of winter tea tree resources to increase production and income for traditional tea farmers. Each acre of tea garden can pick more than 500 kilograms of thick old leaves every year, and about $¥ 4$ per kilogram of original leaves. It is estimated that tea gardens per mu can increase the income of tea farmers by more than $¥ 2000$. In 2018, the area of China's tea gardens reached 43.956 million $\mathrm{mu}$, which is expected to increase the economic income of the entire Chinese tea industry by more than $¥ 80$ billion. This is also in line with the ambitious goal of the Chinese government to resolutely win the fight against poverty by 2020 . Tapping the potential from the tea industry can make full use of your existing resources for the income of tea companies and tea farmers and realize the sustainable development of the tea industry. Through the new process, the thick old leaves in winter are repeatedly twisted and deeply fermented to make "cold bubble" type winter tea. The cold soaking method is very suitable for the tea drinking habits of young people and residents of the "Belt and Road" countries, which is of great significance to the "going out" of China's tea industry.

\section{Conclusion and Discussion}

The existing tea industry lacks innovation capabilities. Most tea farmers and tea 
companies basically follow the traditional ideas and methods for making tea and there is no substantial breakthrough in the development of the tea industry, which makes it difficult for the Chinese tea industry to adapt to the fierce market competition. Winter tea has a new path and makes full use of the thick and old leaves in winter. Not only can it break the bottleneck of the tea making season, but it can also improve the production efficiency of tea gardens, increase the output and output value of tea, be conducive to precision poverty alleviation, extend the industrial chain, and increase the income of tea farmers. It is of great significance to the development of the traditional tea industry. In addition, winter tea has very broad application prospects in biopharmaceuticals, skin care products and food. Winter tea is rich in tea polysaccharides, and can be used directly and indirectly in the treatment of hyperglycemia, hyperlipidemia and other basic drugs, health products and functional food. Therefore, winter tea has higher promotion and application value.

At present, most of the researches on tea have focused on teas such as green tea and black tea, made from buds. The research on tea made from thick old leaves of winter tea trees is relatively few. The main components of winter tea made from old leaves, and the research status of tea polysaccharides, tea polyphenols, and catechins in the main components of winter tea are analyzed. It is believed that winter tea can be used as a raw material for extracting tea polysaccharides to treat hypoglycemia in functional foods and medicines. At the same time, winter tea mainly contains tea polysaccharides, tea polyphenols, and catechins, which have related physiological activities such as improving immunity, antioxidant and anti-cancer. Winter tea powder can also be used as the basis substance for related functional foods, skin care products and drugs. Therefore, this article expands the research on tea, which has certain innovation. However, the method for extracting tea polysaccharides from winter tea has not been studied in detail, and the physiological activities of blood sugar reduction, blood lipids, antioxidant and anti-cancer of winter tea powder have not been verified. These need to be further studied in the future.

\section{Funding}

Research on "Development and Management of New Tea Drink under the Health Theory" funded by Funding Project for Scientific Research Operating Expenses for Universities and Colleagues from University of Science and Technology of China in 2018 (Project No. WK2160000012).

Key research and development project of Anhui Province in 2019: "Development for Winter Tea and its Derivatives” (Project No. 201904a06020014).

\section{Conflicts of Interest}

The authors declare no conflicts of interest regarding the publication of this paper.

\section{References}

Chen, G. J., Yuan, Q. X., Saeeduddin, M. et al. (2016). Recent Advances in Tea Polysac- 
charides: Extraction, Purification, Physicochemical Characterization and Bioactivities. Carbohydrate Polymers, 153, 663-678. https://doi.org/10.1016/j.carbpol.2016.08.022

Chen, J. E., Feng, H. J. et al. (2009). Antioxidant Activity of Active Ingredients in Black Tea, Green Tea and Oolong Tea. Food Science, 30, 62-66.

Chen, X. Q., Lin, Z., Ye, Y., et al. (2010) Suppression of Diabetes in Nonobese Diabetic (NOD) Mice by Oral Administration of Watersoluble and Alkali-Soluble Polysaccharide Conjugates Prepared from Green Tea. Carbohydrate Polymers, 82, 28-33.

Chen, Z. X. (2014). Chinese Tea Dictionary. Beijing: China Light Industry Press.

Crespy, V., \& Wiliamson, G. (2004). A Review of the Health Effects of Green Tea Catechins in in Vivo Animal Models. The Journal of Nutrition, 134, 3431-3440. https://doi.org/10.1093/jn/134.12.3431S

Guo, L. G., Shi, W. M. et al. (2013). Experimental Study on the Prevention and Treatment of Radiation Lung Injury by Tea Catechins. Modern Hospital, 13, 12-14.

He, X. B., Xue, C. K., Wei, S. R. et al. (2007). Inhibition Effect of Tea Polysaccharide on $\alpha$-Amylase Activity and Its Effect on Blood Glucose in Diabetic Rats. Medical Herald, 26, 1284-1286.

Helieh, S., Jeffrey, O., \& Ebersole, L. (2010). Green Tea Polyphenols Mediated Apoptosis Tea Polyphenols Mediated Apoptosis Inintestinal Epithelial Cells Intestinal Epithelial Cells by a FADD-Dependent Pathway Fad-Dependent Pathway. Journal of Cancer Therapy, 1, 105-113. https://doi.org/10.4236/jct.2010.13018

Hou, D. Y., Hui, R. H. et al. (2005). Determination of Polyphenols and Antioxidant Properties of Black Tea. Food Science, No. 8, 367-370.

Hu, X. F., \& Yang, X. Q. (2001). Study on the Effect of Tea Catechins on Apoptosis of Cancer Cells. Tea Science, No. 1, 26-29.

Jiang, Y. W., Hua, J. J. et al. (2018). Analysis and Prospect of China's Black Tea Industry. China Tea Processing, No. 4, 5-10.

Jung, M. H., Seong, P. N., Kim, M. H., Myong, N. H., \& Chang, M. J. (2013). Effect of Green Tea Extract Microencapsulation on Hypertriglyceridemia and Cardiovascular Tissues in High Fructose-Fed Rats. Nutrition Research and Practice, 7, 366-372. https://doi.org/10.4162/nrp.2013.7.5.366

Kao, Y. H., Chang, H. H., \& Lee, M. J. (2006). Tea, Obesity, and Diabetes. Molecular Nutrition \& Food Research, 50, 188-210. https://doi.org/10.1002/mnfr.200500109

Kim, M. J., Nam, H. J., Kim, H. P. et al. (2013). OPB-31121, a Novel Small Molecular Inhibitor, Disrupts the JAK2/STAT3 Pathway and Exhibits an Antitumor Activity in Gastric Cancer Cells. Cancer Letters, 335, 145-152. https://doi.org/10.1016/j.canlet.2013.02.010

Li, S., Li, Y., \& Wang, M. (2003). Study on the Effects of Tea Polyphenols on the Growth Inhibition, Antioxidant and Immune Regulation of Lewis Lung Cancer. Chinese Journal of Cancer Biotherapy, 10, 206-209.

Liu, R. Y., Zeng, Y., Lei, Z. et al. (2014). JAK/STAT3 Signaling Is Required for TGF-Beta-Induced Epithelial-Mesenchymal Transition in Lung Cancer Cells. International Journal of Oncology, 44, 1643-1651. https://doi.org/10.3892/ijo.2014.2310

Ma, L. F., \& Shi, Y. et al. (2003). Analysis of the Content of Inorganic Elements in Famous Green Tea in China and Its Current Status. Tea, No. 2, 83-85.

Mai, Y. L., \& Wang, C. Y. et al. (2019). Study on the Chemical Composition and Quality of Winter Tea. Modern Agricultural Science and Technology, No. 24, 205-206+211.

Martin, M. (2011). The Biology of Aging: 1985-2010 and beyond. The FASEB Journal, 25, 
3756-3762. https://doi.org/10.1096/fj.11-1102.ufm

Peng, H. Q., Cai, W. M., \& Xiang, S. (2003). Effect of Tea Polyphenols against Influenza Virus A3 in Vitro. Tea Science, 23, 19-81.

Ren, T. Y., An, Y. H. et al. (2009). Research Status of Functional Extraction and Health Care Function of Green Tea. Science and Technology of Food and Fermentation, 45, $15-18$.

Sharma, N. H., Phan, H. T. et al. (2020). Black Tea Polyphenol Theaflavin as Promising Antioxidant and Potential Copper Chelator. Journal of the Science of Food and Agriculture. https://doi.org/10.1002/jsfa.10347

Shen, J., Chen, Z. L., Shen, X. Y. et al. (2007). Study on the Anti-Tumor Effect of Tea Polysaccharides and Its Immune Enhancement Effect. Zhejiang Preventive Medicine, 19, $10-12$.

Shi, M., Chen, L. et al. (2018). Research Progress of Tea Polysaccharides in Anti-Oxidation and Delaying Aging. China Aesthetic Medicine, 27, 140-143.

Sun, Y. F., Yang, X. B., Lu, X. S. et al. (2013). Protective Effects of Keemun Black Tea Polysaccharides on Acute Carbon Tetrachloride-Caused Oxidative Hepatotoxicity in Mice. Food and Chemical Toxicology, 58, 184-192. https://doi.org/10.1016/j.fct.2013.04.034

Wang, C. Y., Mai, Y. L., Yan, L. F., Song, W. et al. (2019). Study on the Effect of Different Treatments on the Quality of Winter Tea. Anhui Agricultural Science Bulletin, 25, 124-126.

Wang, D. Y., Zhao, Y., Sun, F. et al. (2014). Protective Effects of Ziyang Tea Polysaccharides on CCl4-Induced Oxidative Liver Damage in Mice. Food Chemistry, 143, 371-378. https://doi.org/10.1016/j.foodchem.2013.08.005

Wang, Y. (2018). Application of Tea Polyphenols in the Food Industry (pp. 395). Abstracts of Papers of the 15th Annual Conference of Chinese Food Science and Technology Society. Chinese Academy of Food Science and Technology.

Wang, Y. F., Peng, Y. H., Wei, X. L. et al. (2010). Sulfationoftea Polysaccharides: Synthesis, Characterization and Hypoglycemic Activity. International Journal of Biological Macromolecules, 46, 270-274. https://doi.org/10.1016/j.ijbiomac.2009.12.007

Wang, Y. L., Zhao, Y., Andrae-Marobela, K. et al. (2013). Tea Polysaccharides as Food Antioxidants: An Old Woman's Tale? Food Chemistry, 138, 1923-1927. https://doi.org/10.1016/j.foodchem.2012.09.145

Yang, B., Huang, W. et al. (2009). Research and Development Status of Green Tea Extracts. Meat Research, No. 12, 88-91.

Yang, J. G., Yu, H. N. et al. (2006). Research Progress on the Effects of Catechins on Prostate Cancer. Chinese Traditional and Herbal Drugs, No. 8, 1275-1278.

Yu, S. C., Liu, C., Wang, C. H. et al. (2012). Antioxidant Activity of Polyphenols in Anji White Tea. Lishizhen Medicine and Materia Medica Research, 23, 1184-1187.

Yu, S. C., Su, T., Yang, J. M., Peng, Z., Lin, J. et al. (2010). Hypoglycemic Effect of Anji White Tea Polysaccharide on Experimental Diabetic Mice. Tea Science, 30, 223-228.

Yu, Z., Shi, Y. T. et al. (2008). New Progress in the Research of Tea Polysaccharides. Guangdong Tea Industry, No. 6, 25-29.

Yuan, Z. Q., Song, W. et al. (2019). Trace Back to the History of Winter Tea and Its Current Development. China Tea, 41, 65-67.

Zhang, X. H., \& Yang, X. Q. (2003) Inhibitory Effect of Tea Polyphenols and Catechins on the Growth of Prostate Cancer Cells. Tea, 29, 142-144. 
Zhang, Y., Ni, D. J., Chen, Y. B. et al. (2011). Study on the Effect of Oolong Tea Polysaccharide on Blood Lipid Regulation and Its Mechanism. Tea Science, 31, 399-404.

Zhao, B. L. (2008). Tea Polyphenols Protect the Brain Nerves from Parkinson's Disease Damage and Its Molecular Mechanism. Progress in Biochemistry and Biophysics, No. 7, 735-743.

Zheng, Q., Ding, L. et al. (2005). Study on the Effect of Catechin Catechins on Inhibiting the Proliferation of Influenza Virus. Public Health and Preventive Medicine, No. 4, 12-14.

Zhou, B. X., Kong, L. B., Chen, J. X. et al. (2009). Extraction of Polysaccharides from Pu'er Tea and Study on Hypoglycemic. Chinese Agricultural Science Bulletin, 25, 55-59.

Zhu, C., Zhao, S. H. et al. (2015). Research Status and Prospects of Black Tea Fermentation Process. Anhui Agricultural Sciences, 43, 383-384+387.

Zhu, Z. Q., Lu, D. B. et al. (2008). Current Status of Green Tea Industry Development in China. China Tea, No. 9, 4-5. 